\title{
Modelling and control of fouling in submerged membrane bioreactor using neural network internal model control
}

\author{
Nurazizah Mahmod ${ }^{1}$, Norhaliza Abdul Wahab ${ }^{2}$, Muhammad Sani Gaya ${ }^{3}$ \\ ${ }^{1,2}$ School of Electrical Engineering, Faculty of Engineering, Universiti Teknologi Malaysia, 81310 Skudai, \\ Johor, Malaysia \\ ${ }^{3}$ Kano University of Science and Technology, Wudil \& Faculty of Electrical Engineering, Nigeria
}

\begin{tabular}{l} 
Article Info \\
\hline Article history: \\
Received Nov 14, 2019 \\
Revised Jan 28, 2020 \\
Accepted Feb 11, 2020 \\
\hline
\end{tabular}

\section{Keywords:}

ANN

FFNN

Fouling

Internal model control

Membrane bioreactor

NARXNN

RBFNN

\begin{abstract}
Membrane bioreactor (MBR) is one of the best solutions for water and wastewater treatment systems in producing high quality effluent that meets its standard regulations. However, fouling is one of the main issues in membrane filtration for membrane bioreactor system. The prediction of fouling is crucial in the membrane bioreactor control system design. This paper presents an intelligence modeling system so called artificial neural network (ANN). The feedforward neural network (FFNN), radial basis function neural network (RBFNN) and nonlinear autoregressive exogenous neural network (NARXNN) are applied to model the submerged MBR filtration system. The simulation results show good predictions for all methods which the highest performance of the model given by RBFNN. Based on the developed models, the neural network internal model control (NNIMC) is implemented to control fouling development in membrane filtration process. Three different control structures of the NNIMC are proposed. The FFNN IMC, RBFNN IMC and NARXNN IMC controllers are compared to the conventional IMC. The RBFNN IMC has a superior performance both in tracking and disturbance rejections.
\end{abstract}

This is an open access article under the CC BY-SA license.

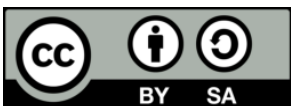

Corresponding Author:

Norhaliza Abdul Wahab,

School of Electrical Engineering, Faculty of Engineering,

Universiti Teknologi Malaysia,

81310 Skudai, Johor, Malaysia.

Email: aliza@fke.utm.my

\section{INTRODUCTION}

High concern on the quality of water and wastewater has led to more research on treatment technology. Membrane bioreactor (MBR) is one of the technologies to replace conventional activated sludge (CAS) because MBR can be classified as the best alternative solution. Several advantages have been discovered by using MBR system over conventional technologies [1]. The system has been verified to be very competent since it able to give better treatment in wastewater in term of producing good and high quality of effluent. The MBR technology is a combination of biological process and filtration system. The membrane filtration system is a complex process and hard to be modelled. The filtration systems still struggling from many issues such as fouling and energy efficiency [2-5]. Fouling can be determined as undesirable of the accumulation of substances such as colloidal, particulate, solute material and microorganism on the membrane during filtration process [6]. Fouling can cause blockage of membranes where the membrane pore will be blocked by solid material. When this phenomenon occurs, the permeate flux will decline and cause rapid increment in transmembrane pressure (TMP). Fouling also caused the 
increment of hydraulic resistance in the filtration system and if not controlled will cause to membrane damage.

The development of reliable prediction model for membrane filtration system is important to improve the performance of the system [7-8]. To avoid flux decline in the MBR filtration process, a reliable feedback control method is needed. This method requires an acceptable degree of model accuracy and model reliability to ensure the controller is at the desired performance. Intelligence modeling technique which is artificial neural network (ANN) is used to model the developed submerged MBR filtration system [9]. ANN is widely used and among the most popular algorithm for modeling membrane filtration system. Three different structures of ANN such as radial basis function neural network (RBFNN), multilayer perceptron neural network and general regression neural network have been used in modeling of filtration system for synthetic wastewater treatment [10]. All three structures give better prediction of the TMP profile during the process of filtration. Work by [11] applied the RBFNN to model the effluent quality parameters of an SMBR treating combined municipal and industrial wastewater. The best results showed that the testing and training procedure by RBFNN were successful. The RBFNN has also been successfully applied for solving dynamic system problems in [12-13] and it can predict the dynamic behaviour directly from input and output data. In addition, in [14] developed two types of feedforward artificial network which are multilayer perceptron and radial basis function to achieve of this work. The selection of radial basis function and multilayer perceptron are because of their ability to detect complex nonlinear relationships in the data, representing two different approaches to solving problems. The result show that ANN technique have a good performance and high accuracy. Work by [15-16] demonstrated RBFNN to predict the interfacial interactions with randomly rough membrane surface. The proposed RBFNN method has broad application prospects in membrane fouling and interface behaviour research. The robust RBFNN proposed paved a new way to study membrane fouling in MBRs.

In process control, internal model control (IMC) is often used due to its simplicity and good disturbance elimination. The application of IMC controller has been established in many industrial applications. IMC techniques belong to a very simple, easy to implement, robust, and suitable for many industrial applications [17]. IMC also success implemented in waste water treatment system but not yet implemented in submerged membrane bioreactor filtration process. The IMC algorithm is effective in rejecting disturbances and achieving good performance. IMC has shown greater capability in control of complex systems compared to the classical control techniques [18]. Previous works shows that the combination of ANN with IMC provide better performance of control system. In [19] proposed neural network internal model control (NNIMC) to control the top and bottom composition in distillation column. The result showed NNIMC provide better performance when compared to the conventional proportional integral plus feedforward controller. Work by [20] applied NNIMC using feed forward architecture to control bioreactor composition both for set point and disturbance rejection. In [21-22] the recurrent neural network architecture is used to train the inverse model controller in IMC configuration. The results showed that the NNIMC has better performance than the optimally tuned linear controllers for setpoint and disturbance rejection in a variety of conditions. Furthermore, the application of IMC can also demonstrated in [23] for simulation of inverted pendulum. IMC was designed using the inverse of the plant model in series with a low-pass filter. The results shows robustness of the IMC and got graded responses when compared with PID. In [24] implemented IMC and neuro-fuzzy IMC (NFIMC) for control of nonlinear process was designed to control of a thermo-optical plant. The main aim is to show the effectiveness of the proposed algorithm. The NFIMC was design modified IMC scheme to increase the disturbance rejection and the robustness performance. The results showed that the NFIMC has better performance and very effective method.

As for this paper, it is consist of four main sections and are organized as follows. The first section started with the background of this study and several literature on the related works. Then, second section presents the methodology of neural network model development and IMC controller design for submerged MBR filtration process while in the third section is the results and discussion. Finally, the forth section gives conclusion and future works based on this study.

\section{RESEARCH METHOD}

Successful operation of wastewater treatment plant depends on the accuracy of the models for control system development. Several nonlinear intelligence modelling techniques which are feedforward neural network (FFNN), RBFNN and nonlinear autoregressive exogenous neural network (NARXNN) were performed for modeling of MBR system. The input is permeate pump voltage and and the outputs are permeate flux and transmembrane pressure of filtration processes. The performance of the models is measured using three criteria which are correlation coefficient $\left(\mathrm{R}^{2}\right)$, mean square error and absolute deviation. For control part, the NNIMC was implemented to control fouling development in membrane 
filtration process. The performance of the controller was measured using rise time, settling time, overshoot, IAE, ISE and ITAE. The details of the flow of methodology is depicted in Figure 1.

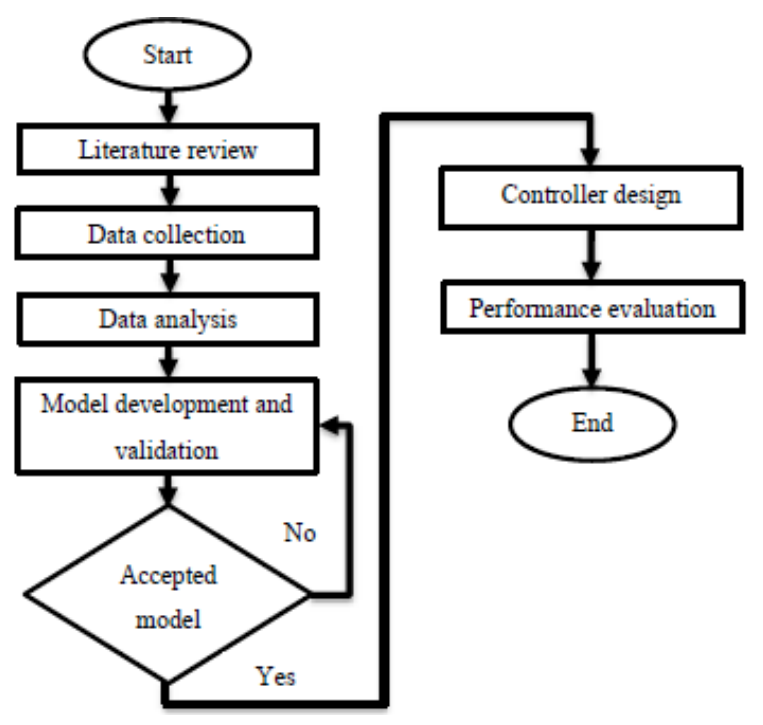

Figure 1. Flow of research methodology

In this work, the filtration data sets were collected from the developed SMBR pilot plant. Steps input with random magnitude were excited into the suction pump to obtain the dynamic behaviour of the filtration process. During the operation, permeate to relaxation period is maintained at 120 seconds permeate and 30 seconds relaxation was chosen with continuous aeration airflow. The aeration airflow during filtration is set around 6 to 8 SLPM to maintain high intensity of bubble flow in cleaning the membrane. The input of this filtration process is the permeate pump voltage while the outputs are the flux and transmembrane pressure (TMP). The SMBR filtration data set is shown in Figure 2. From the data, it can be observed that the permeate flux and TMP has an impact with the changes of permeate pump voltage setting. At the high voltage setting, permeate flux and TMP is higher than at the low voltage setting.

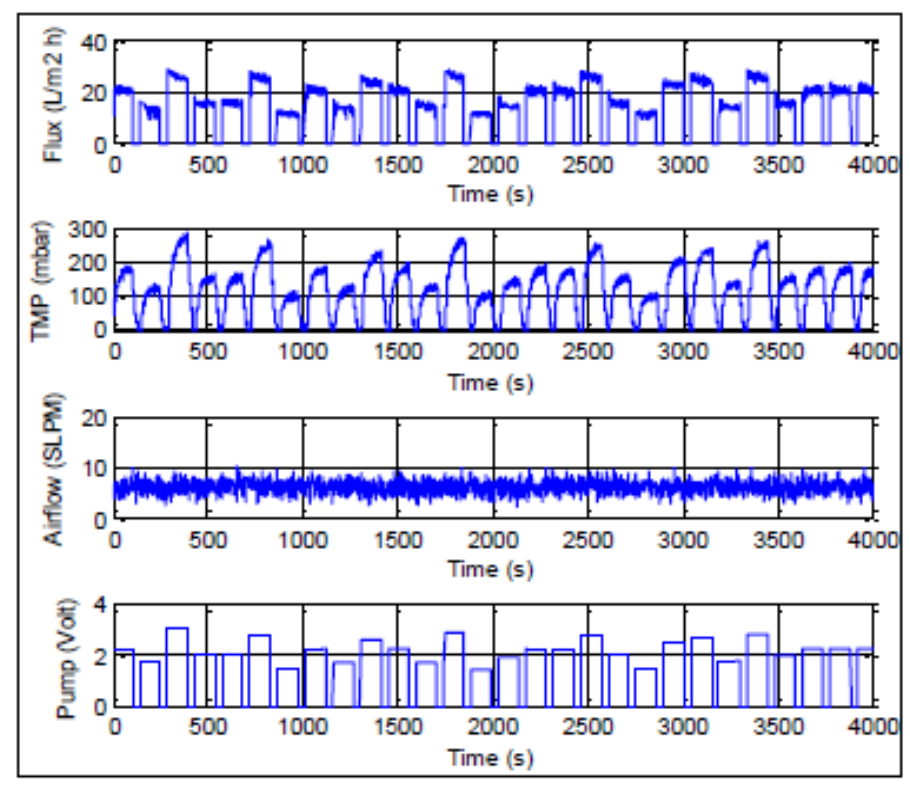

Figure 2. Input and outputs SMBR filtration process 


\subsection{Neural network model}

The network architecture with the best hidden neuron, learning rate value and spread value were used to train the acquired set of 2400 data. The MBR filtration data are divided into $60 \%$ and $40 \%$ for training and testing data set, respectively. The inverse of the developed FFNN, RBFNN and NARXNN models are then trained, where voltage as an output data and flux and TMP as inputs data using LevenbergMarquard learning algorithm with back propagation. To improve robustness, the effects of mismatch between the actual and the predicted model was minimized. The differences between the actual data and the predicted model occurred at high frequency response of the system, a low-pass filter is thus added to attenuate this effect [25]. In this case, IMC is designed for the aforementioned ANN structures using the inverse of the predicted model in series with a low-pass filter. The low pas filter had been used to filter high frequency signal and adjusting the controller sensitivity. For membrane fouling control, the second order filter is identified with the filter tuning parameter of 0.001 . The filter transfer function, $G_{f}(\mathrm{~s})$ for the system is [25]:

$$
G_{f}(s)=\frac{1}{(0.001 s+1)^{2}}
$$

\subsection{Neural network internal model control}

The developed ANN models are applied for IMC controller design. In this work, the NNIMC is designed such that to improve the performance of disturbance rejection through disturbance feedback signal and filtering action as shown in Figure 3. In this paper, the inverse model were obtained by inverting the neural network model using Newtons method and solving numerically the control action by directly trained inverse model neural network controller [26]. The direct trained neural network has been utilized in this work to design the neural network controller and show good performance both for set point and disturbance rejection. The designed filters able to improve disturbance rejection and set point tracking and this requires good understanding of the behaviour of the system for the selection of filtering tuning parameter. The comparison between the three ANN based IMC control structures namely FFNN-IMC, RBFNN-IMC and NARXNN-IMC are presented. The robustness issue is confirmed by stability of the system with respect to disturbance rejection performance. While, the best performance of modelling which is RBFNN was used to design the conventional-IMC to control fouling development in membrane filtration process.

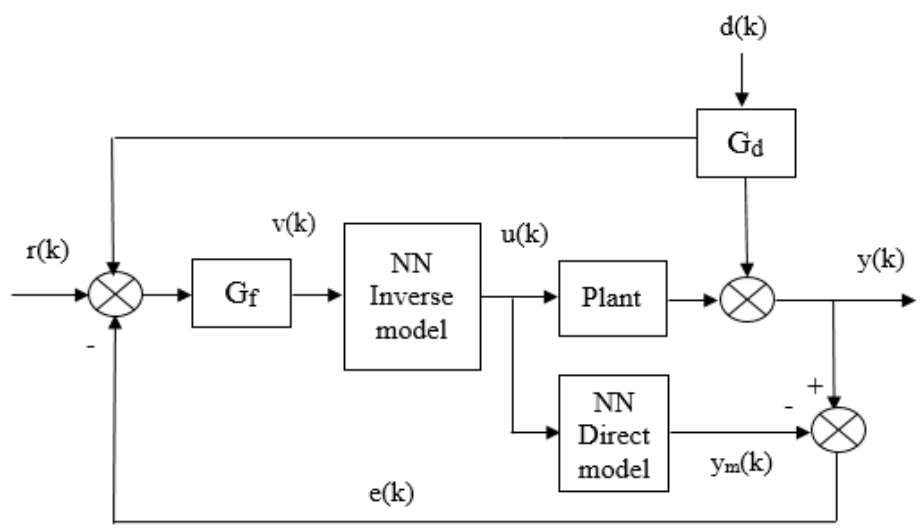

Figure 3. The NNIMC block diagram

\section{RESULTS AND DISCUSSION}

The submerged MBR filtration data are divided into training and testing data set which is $60 \%$ for training and $40 \%$ for testing. The input parameter is permeate pump voltage and the outputs are the permeate flux and TMP of the filtration process. The performance of the models was evaluated using three criteria which are correlation coefficient $R^{2}$, mean square error (MSE) and absolute deviation (MAD).

\subsection{Submerged MBR filtration training and testing}

The training and testing results of the flux and TMP models are plotted as shown in Figure 4 (a)-(b) and Figure 5 (a)-(b), respectively. The comparison between FFNN, RBFNN and NARXNN model structures with the actual data were discussed. For the training of the flux, almost similar performance observed from all structures with $\mathrm{R}^{2}$ of $90.15 \%, 90.55 \%$ and $90.39 \%$, respectively. The MSE give, of respectively 0.0057 , 
0.0055 and 0.0056 for FFNN, RBFNN and NARXNN. The MAD values for FFNN and RBFNN are 0.0446 and 0.0440 and 0.0444 for NARXNN. In terms of number of neuron, the RBFNN requires only three hidden neurons compared with FFNN and NARXNN which is five and six hidden neurons, respectively. For TMP, the RBFNN demonstrated the best model as compared to FFNN and NARXNN with value of $\mathrm{R}^{2}$ is $91.29 \%$ and MSE value of 0.0050 . For the training result, the RBFNN is more accurate and reliable compared with the other techniques.

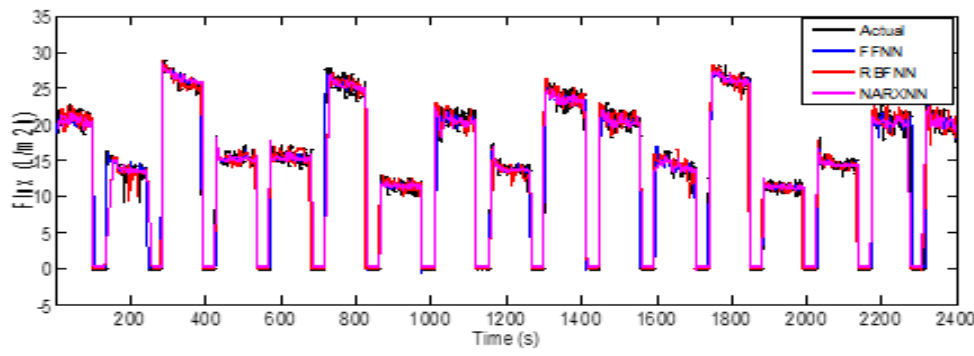

Figure 4. (a) Flux model training

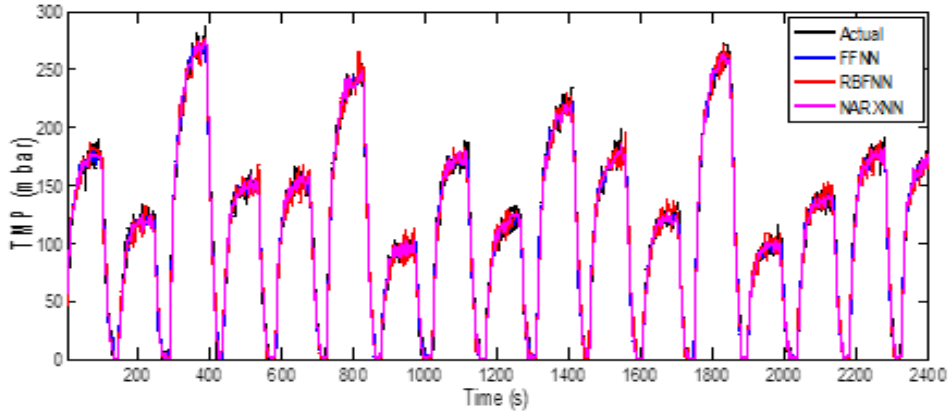

Figure 4. (b) TMP model training

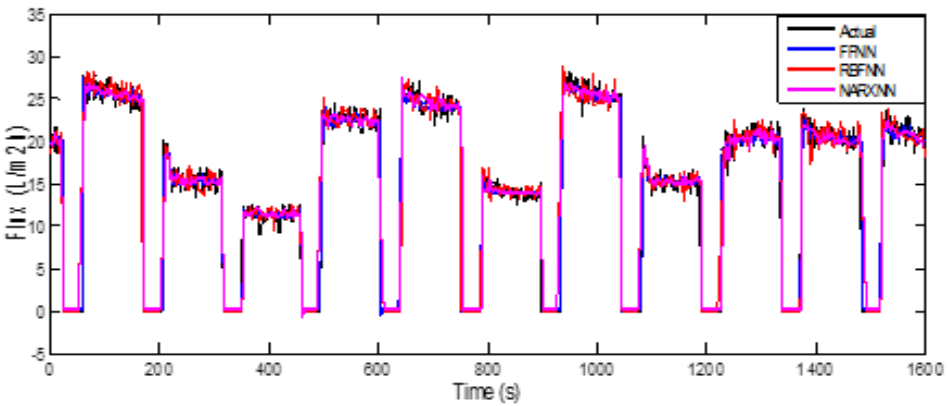

Figure 5. (a) Flux model testing

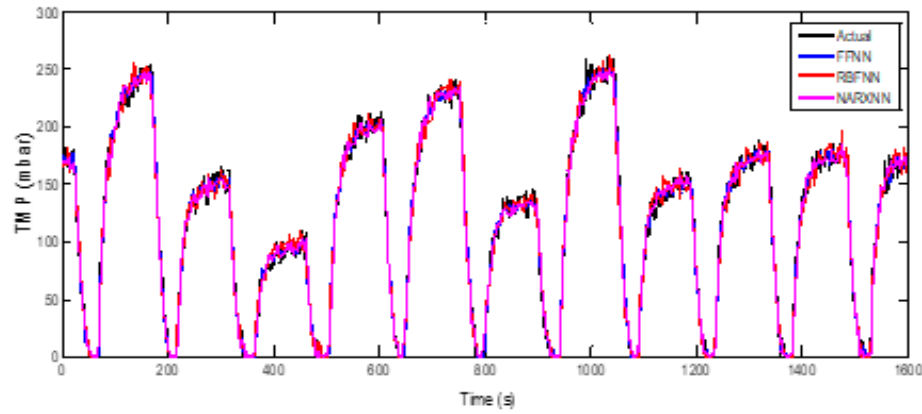

Figure 5. (b) TMP model testing 
From the testing results in Figure 5 (a)-(b), slightly similar trend of performance was depicted as given by the training results. The RBFNN showed superior performance for both flux and TMP compared to the other two methods in term of $\mathrm{R}^{2}$, MSE and MAD. Tables 1 and 2 provide more details results for the flux and TMP, for the training and testing, respectively.

Table 1. Flux and TMP training results

\begin{tabular}{cccc}
\hline Flux Models & $\mathrm{R}^{2}$ & MSE & MAD \\
\hline FFNN & 90.15 & 0.0057 & 0.0446 \\
RBFNN & 90.55 & 0.0055 & 0.0440 \\
NARXNN & 90.39 & 0.0056 & 0.0444 \\
\hline TMP Models & $\mathrm{R}^{2}$ & MSE & MAD \\
\hline FFNN & 88.43 & 0.0061 & 0.0543 \\
RBFNN & 91.29 & 0.0050 & 0.0340 \\
NARXNN & 90.34 & 0.0059 & 0.0476 \\
\hline
\end{tabular}

Table 2. Flux and TMP Modelling testing results

\begin{tabular}{cccc}
\hline Flux Models & $\mathrm{R}^{2}$ & MSE & MAD \\
\hline FFNN & 90.22 & 0.0063 & 0.0446 \\
RBFNN & 92.45 & 0.0050 & 0.0343 \\
NARX NN & 92.33 & 0.0052 & 0.0365 \\
\hline TMP Models & $\mathrm{R}^{2}$ & MSE & MAD \\
\hline FFNN & 88.67 & 0.0061 & 0.0564 \\
RBFNN & 91.11 & 0.0051 & 0.0230 \\
NARXNN & 91.32 & 0.0053 & 0.0233 \\
\hline
\end{tabular}

\subsection{Neural network internal model control}

In this section, the NNIMC with different structures namely FFNN IMC, RBFNN IMC and NARXNN is compared with the conventional IMC (C-IMC) both for tracking and disturbance rejection performances. Figure 6 (a)-(c) show the input and output responses for all controller for tracking perperfomance. The performance of the controller was measured using several criteria as shown in Table 3. The step response performance of the controller is at $20 \mathrm{~L} / \mathrm{m}^{2} \mathrm{~h}$ of permeate flux. The percentage of IAE showed the lowest for RBFNN IMC with 0.0422 , followed by 0.0631 for NARXNN IMC, 0.0785 for FFNN IMC and 0.1178 for C-IMC. The fastest settling time is given by RBFNN IMC with only 3.75 seconds which is more than $80 \%$ faster than the C-IMC. Overall, the RBFNN IMC showed the best tracking performance in almost all criteria as shown in Table 3 and hence is preferable to other methods.

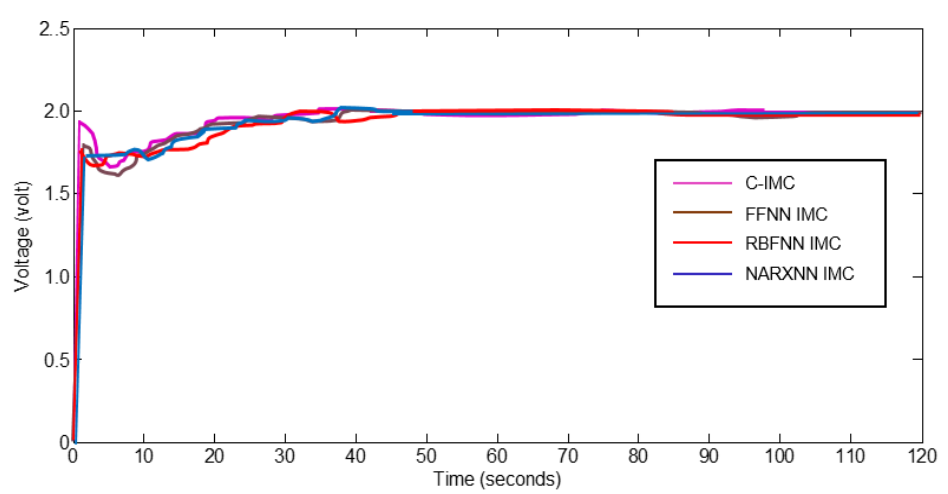

(a)

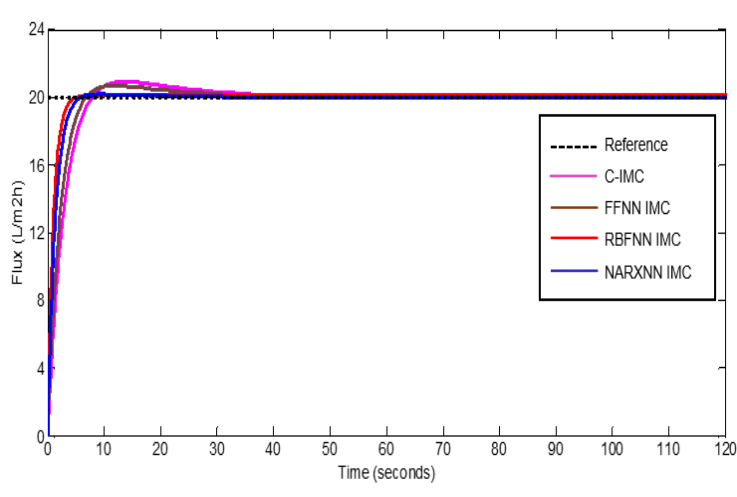

(b)

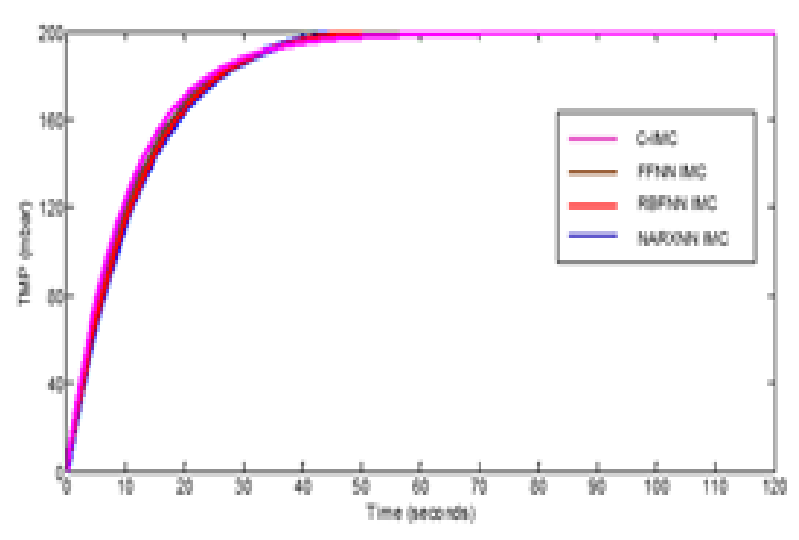

(c)

Figure 6. (a) Voltage input response; (b) Flux output response; (c) TMP output response 
Table 3. Tracking performance index for all controllers

\begin{tabular}{ccccc}
\hline Criteria & C-IMC & FFNN IMC & RBFNN IMC & NARXNN IMC \\
\hline Rise time (s) & 5.86 & 4.53 & 1.51 & 2.04 \\
Settling time & 27.51 & 25.77 & 3.75 & 4.52 \\
Overshoot (\%) & 10.45 & 6.73 & 2.44 & 3.98 \\
IAE & 0.1178 & 0.0785 & 0.0422 & 0.0631 \\
ISE & 0.2913 & 0.0083 & 0.0024 & 0.0054 \\
ITAE & 0.4245 & 0.0906 & 0.0599 & 0.0675 \\
\hline
\end{tabular}

Figures 7 (a)-(d) show the amplitude of step disturbances and flux performances for disturbance rejections. The performance of C-IMC, FFNN IMC, RBFNN IMC and NARXNN IMC controllers are plotted as shown in Figure 7. The step disturbances of magnitude $10 \%$ of the flux for 20 seconds was excited first into the system and the performance of the system is observed. As seen in Figure 7(b), the RBFNN IMC provides small overshoot for $10 \%$ amplitude of disturbance rejection compared to other methods. To see the robustness of the proposed controller, 50\% disturbance was also excited into the system. The RBFNN IMC remains the highest performance in disturbance rejection, followed by the NARXNN and FFNN IMC controllers as presented in Figure 7(d). The C-IMC depicted the highest overshoot in respond to the disturbance.

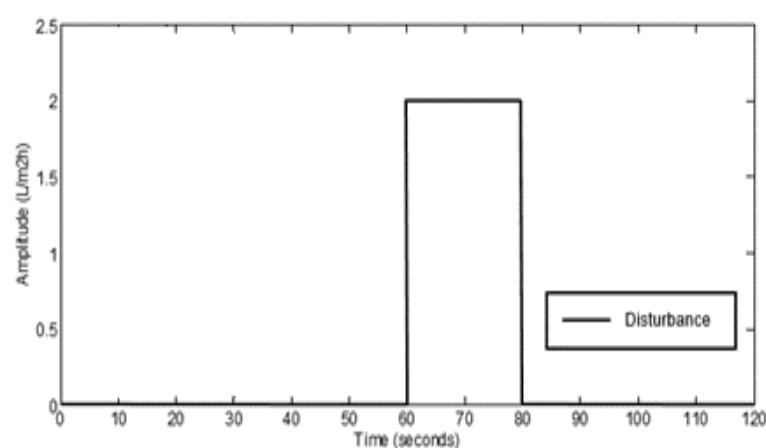

(a)

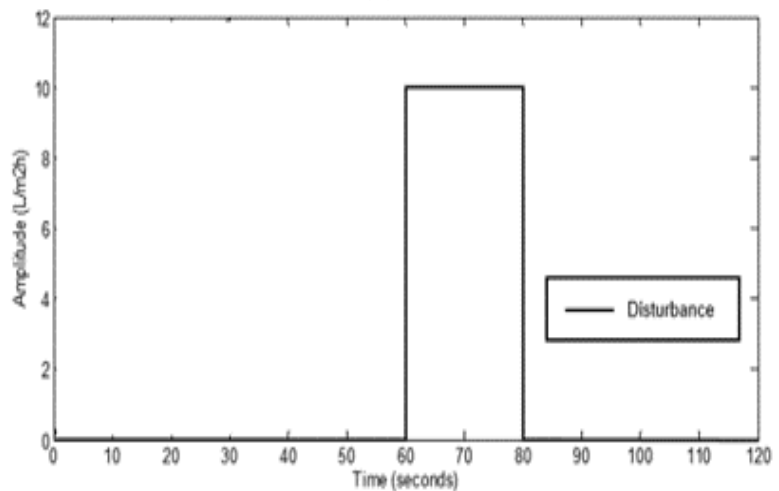

(c)

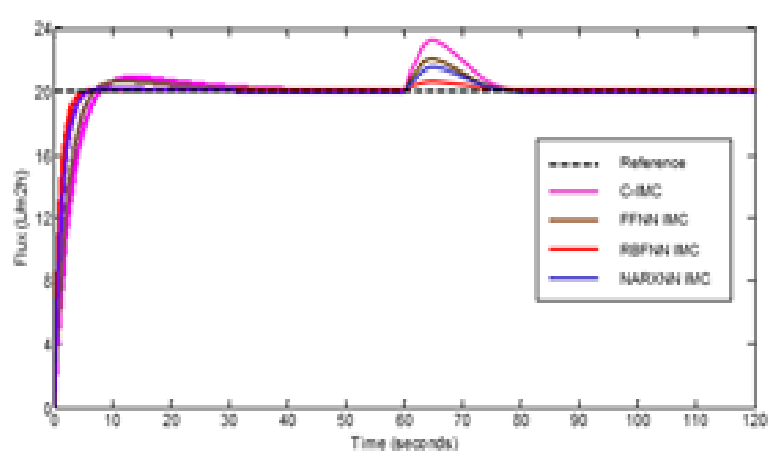

(b)

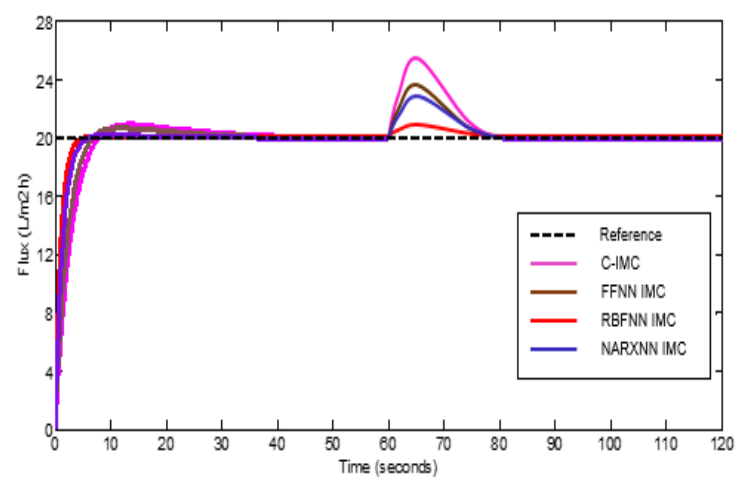

(d)

Figure 7. (a) 10\% disturbance rejection; (b) Flux performance for 10\%; (c) 50\% disturbance rejection; (d) Flux performance for $50 \%$;

\section{CONCLUSION}

Modelling and control is an important aspect of the membrane bioreactor filtration system. Obtaining a reliable model is a very challenging task because of the nature of the process and fouling phenomenon. However, good filtration process is crucial and hence good model for control system design is needed. Three prediction models for submerged MBR system was developed which is the FFNN, RBFNN and NARXNN models. From the simulation results, it can be observed that the RBFNN and NARXNN give good results for flux and TMP prediction models compared to FFNN. However, the RBFNN is preferable due to high accuracy and more reliable prediction compared with other two methods. For the control part, three structures of NNIMC have been developed and compared to the conventional IMC. The results showed 
the RBFNN IMC provides better tracking performance than FFNN IMC, NARXNN IMC and C-IMC for all performance criteria. In addition, the RBFNN IMC performed well in 10\% disturbance rejection compared to other methods. It is also seen to be more robust when able to remains with low overshoot for the 50\% disturbance rejection.

Several recommendations of work can be considered for further improvement. In modelling of SMBR, an external optimisation such as particle swarm optimisation and gravitational search algorithm can be implemented to enhance the prediction of the filtration models. Other nonlinear modelling technique such as wavelet neural network can be applied to predict the behaviour of long term filtration cycle. In the controller design part, other type of disturbance test can be applied to establish the reliability of the controller in handling disturbances which can reveal the performance of the controller under mismatch model conditions

\section{ACKNOWLEDGEMENTS}

The authors would like to thank the Ministry of Education (MOE) PRGS vote R.J130000.7851.4L702 and the Universiti Teknologi Malaysia UTMHIR vote 08G74 for their financial and facility support of this work.

\section{REFERENCES}

[1] T. Stephenson, K. Brindle, S. Judd, and B. Jefferson, Membrane bioreactors for wastewater treatment: IWA publishing, 2000.

[2] A. Drews, "Membrane fouling in membrane bioreactors - characterisation, contradictions, cause and cures," Journal of membrane science, vol. 363, pp. 1-28, 2010.

[3] P. Le-Clech, V. Chen, and T. A. Fane, "Fouling in membrane bioreactors used in wastewater treatment," Journal of membrane science, vol. 284, pp. 17-53, 2006.

[4] S. Judd, The MBR book: principles and applications of membrane bioreactors for water and wastewater treatment: Elsevier, 2010.

[5] Z. Wang, J. Ma, C. Y. Tang, K. Kimura, Q. Wang, and X. Han, "Membrane cleaning in membrane bioreactors: a review," Journal of membrane science, vol. 468, pp. 276-307, 2014.

[6] E. H. Bouhabila, R. B. Aïm, and H. Buisson, "Fouling characterisation in membrane bioreactors," Separation and Purification Technology, vol. 22, pp. 123-132, 2001.

[7] K. Hunt and D. Sbarbaro, "Neural networks for nonlinear internal model control," in IEE Proceedings D (Control Theory and Applications), 1991, pp. 431-438.

[8] R. W. Okey, "Phosphorous removal in membrane waste water treatment," ed: Google Patents, 1968.

[9] A. R. Pendashteh, A. Fakhru'l-Razi, N. Chaibakhsh, L. C. Abdullah, S. S. Madaeni, and Z. Z. Abidin, "Modeling of membrane bioreactor treating hypersaline oily wastewater by artificial neural network," Journal of hazardous materials, vol. 192, pp. 568-575, 2011.

[10] L. Erdei, S. Vigneswaran, and J. Kandasamy, "Modelling of submerged membrane flocculation hybrid systems using statistical and artificial neural networks methods," Journal of Water Supply: Research and Technology-Aqua, vol. 59, pp. 198-208, 2010.

[11] S. A. Mirbagheri, M. Bagheri, S. Boudaghpour, M. Ehteshami, and Z. Bagheri, "Performance evaluation and modeling of a submerged membrane bioreactor treating combined municipal and industrial wastewater using radial basis function artificial neural networks," Journal of Environmental Health Science and Engineering, vol. 13, p. 17, 2015.

[12] S. Ferrari, F. Bellocchio, V. Piuri, and N. A. Borghese, "A Hierarchical RBF Online Learning Algorithm for RealTime 3-D Scanner," IEEE Transactions on Neural Networks, vol. 21, pp. 275-285, 2010.

[13] H.-G. Han and J.-F. Qiao, "Prediction of activated sludge bulking based on a self-organizing RBF neural network," Journal of Process Control, vol. 22, pp. 1103-1112, 2012.

[14] M. Bagheri, S. A. Mirbagheri, A. M. Kamarkhani, and Z. Bagheri, "Modeling of effluent quality parameters in a submerged membrane bioreactor with simultaneous upward and downward aeration treating municipal wastewater using hybrid models," Desalination and Water Treatment, vol. 57, pp. 8068-8089, 2016/04/14 2016.

[15] Z. Zhao, Y. Lou, Y. Chen, H. Lin, R. Li, and G. Yu, "Prediction of interfacial interactions related with membrane fouling in a membrane bioreactor based on radial basis function artificial neural network (ANN)," Bioresource Technology, vol. 282, pp. 262-268, 2019/06/01/2019.

[16] Y. Chen, G. Yu, Y. Long, J. Teng, X. You, B.-Q. Liao, et al., "Application of radial basis function artificial neural network to quantify interfacial energies related to membrane fouling in a membrane bioreactor," Bioresource Technology, vol. 293, p. 122103, 2019/12/01/ 2019.

[17] T. Liu, F. Gao, and Y. Wang, "IMC-based iterative learning control for batch processes with uncertain time delay," Journal of Process Control, vol. 20, pp. 173-180, 2010.

[18] G. C. Goodwin, S. F. Graebe, and M. E. Salgado, "Control system design," Upper Saddle River, p. 13, 2001.

[19] D. Muhammad, Z. Ahmad, and N. Aziz, "Implementation of internal model control (imc) in continuous distillation column," in Proceedings of the 5th International Symposium on Design, Operation and Control of Chemical Processes, 2010, pp. 812-821. 
[20] I. Hajizadeh and S. Hosseini, "Artificial Neural Networks Based Control of CSTRs with Van de Vusse Reaction," Intelligent Automation and Soft Computing, 10/31 2014.

[21] M. A. Hussain, "Review of the applications of neural networks in chemical process control—simulation and online implementation," Artificial intelligence in engineering, vol. 13, pp. 55-68, 1999.

[22] M. Nikolaou and V. Hanagandi, "Control of nonlinear dynamical systems modeled by recurrent neural networks," AIChE journal, vol. 39, pp. 1890-1894, 1993.

[23] D. J. Vora and M. N. Basu, "Modelling and Simulation of Inverted Pendulum using Internal Model Control," Journal for Research $\mid$ Volume, vol. 1, 2016.

[24] J. Paulusová and M. Paulus, "Internal model control of thermo-optical plant," in Process Control (PC), 201721 st International Conference on, 2017, pp. 179-184.

[25] W. R. M. Issa, "A New Approach of Robust Internal Model Control for Nonlinear Pendulum System," A New Approach of Robust Internal Model Control for Nonlinear Pendulum System, 2011.

[26] M. A. Henson and D. E. Seborg, "An internal model control strategy for nonlinear systems," AIChE Journal, vol. 37, pp. 1065-1081, 1991.

\section{BIOGRAPHIES OF AUTHORS}

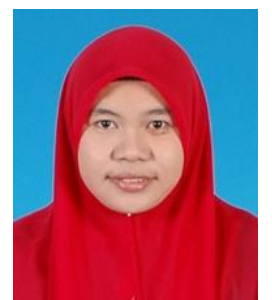

Nurazizah Binti Mahmod is Master student in School of Electrical Engineering, Faculty of Engineering, Universiti Teknologi Malaysia, Skudai, Johor. Received her bachelor degree in Electrical Control \& Instrument in Universiti Teknologi Malaysia, Skudai, and Johor. Her area of interest is applying data mining technique in Wastewater Treatment fields and application.

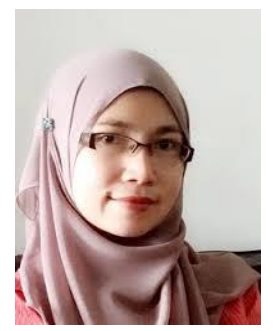

Ir. Dr Norhaliza Abdul Wahab is currently an Associate Professor at Universiti Teknologi Malaysia (UTM). She is currently the Director for Control and Mechatronic Engineering at the School of Electrical Engineering, UTM. She completed her PhD in Electrical Engineering majoring in Control in July 2009. She is actively involved in researching and teaching in the field of industrial process control. Her expertise is in modelling and control of industrial process plant. Recently she has worked primarily on different types of domestic and industrial water and wastewater treatment technology towards optimization and energy saving system.

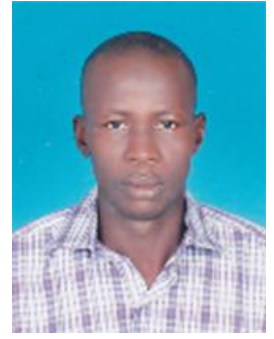

Muhammad Sani Gaya received B.Eng in Electrical Engineering in 2002, M.Eng (Electrical Engineering.) in 2008 both from Bayero University Kano, Nigeria and PhD degree from the Department of Control and Mechatronics Engineering, Universiti Teknologi Malaysia in 2014. His current research interest centres around system identification, feedback control, IMC and inverse control of uncertain/ nonlinear systems based on neural network, fuzzy and ANFIS. Currently, working with Department of Electrical Engineering, Kano University of Science and Technology, Wudil. 